\title{
Using Simulation Results to Choose a Latent Trait Model
}

\author{
Wendy M. Yen \\ CTB/McGraw-Hill
}

A latent trait model goodness-of-fit statistic was defined, and its relationships to several other commonly used fit statistics were described. Simulation data were used to examine the behavior of these fit statistics under conditions similar to those found with real data. The simulation data were generated for 36 pseudo-items and 1,000 simulees using three-, two-, and one-parameter logistic latent trait models. The data were analyzed using three-, two-, and one-parameter models. Between-model comparisons were made of the fit statistics, trait es- timates, and item parameter estimates. The three generating models produced clearly different patterns of results. The simulation results were compared to results for real data involving seventh- and eighth-grade students' performance on eight achievement tests. The achievement test results appeared most similar to the simulation results based on data generated with the three-parameter model. Some practical problems that can result from using an inappropriate model with multiple-choice tests are discussed.

There are three latent trait models that are in common use, and they differ in terms of the number of item parameters they estimate to describe item characteristic functions. The three-parameter (3-PAR) model estimates item difficulties, discriminations, and lower asymptotes. The two-parameter (2-PAR) model estimates item difficulties and discriminations but assumes that all lower asymptotes are zero. The one-parameter (1-PAR), or Rasch, model estimates item difficulties but assumes that all item discriminations are constant and all lower asymptotes are zero. (See Allen \& Yen, 1979; Lord \& Novick, 1968; or Journal of Educational Measurement, Summer 1977, for a further discussion of these models.) When items have a multiple-choice format and it is possible for examinees of low ability to get an item correct through lucky guessing, the 2-PAR and 1-PAR models appear a priori to be inappropriate. However, in striving for simplicity, the researcher may be tempted to use the 2-PAR or 1-PAR model and hope that the inaccuracies that are introduced are unimportant. The researcher may turn to a statistical goodness-of-fit test to gauge the degree of these inaccuracies.

This paper examines the behavior of a fit statistic, called $Q_{1}$, which is similar to fit statistics that are commonly used for latent trait models. In the following sections (1) $Q_{1}$ is specified, (2) informal theoretical justification is given for using a chi-square distribution as an approximation to the distri- 
bution of $Q_{1}$ when the latent trait model being tested is true, and (3) the relationships of $Q_{1}$ to some other measures of fit are defined. A simulation study is conducted to examine the behavior of $Q_{1}$ and two other fit statistics under conditions similar to those found with real data. The usefulness of $Q_{1}$ in choosing the most appropriate latent trait model for a set of data is examined; and an additional graphical method of examining the suitability of the three latent trait models also is presented. Finally, real-data examples are presented of how the fit measure, $Q_{1}$, and the graphical comparisons were used in helping choose an appropriate latent trait model for several achievement tests.

\section{Measures of Fit in Latent Trait Theory}

\section{$Q_{1}$ : Definition}

$Q$, is a fit statistic that is similar to the fit measures commonly used to examine latent trait models. To calculate $Q_{1}$, examinees are rank ordered on the basis of their trait estimates and then divided into 10 cells with approximately equal numbers of examinees per cell. The value of the fit statistic for item $i$ is

$$
\begin{aligned}
Q_{1 i} & =\sum_{j=1}^{10} \frac{N_{j}\left(O_{i j}-E_{i j}\right)^{2}}{E_{i j}} \\
& +\sum_{j=1}^{10} \frac{N_{j}\left[\left(1-O_{i j}\right)-\left(1-E_{i j}\right)\right]^{2}}{1-E_{i j}} \\
& =\sum_{j=1}^{10} \frac{N_{j}\left(O_{i j}-E_{i j}\right)^{2}}{E_{i j}\left(1-E_{i j}\right)},
\end{aligned}
$$

where

$N_{j}$ is the number of examinees in cell $j$,

$O_{i j}$ is the observed proportion of examinees in cell $j$ that passes item $i$, and

$E_{\imath}$ is the predicted proportion of examinees in cell $j$ that passes item $i$.

$$
E_{i j}=\frac{1}{N_{j}} \underset{k \in j}{N} \hat{P}_{i}\left(\hat{O}_{k}\right),
$$

where $\hat{P}_{i}\left(\hat{\theta}_{k}\right)$ is the item characteristic function for item $i$, which is evaluated using the trait estimate for examinee $k, \hat{\theta}_{k}$, and the item parameters estimated for item $i$. The summation is taken over examinees in cell $j$.

$$
\hat{P}_{i}\left(\hat{O}_{k}\right)=\hat{c}_{i}+\frac{1-\hat{c}_{i}}{1+e^{-1.7 \hat{a}_{i}\left(\hat{\theta}_{k}-\hat{b}_{i}\right)}} \text {. }
$$

The terms $\hat{a}_{i}, \hat{b}_{i}$, and $\hat{c}_{i}$ are, respectively, the estimated discrimination, difficulty, and lower asymptote for item $i$. For the 2-PAR model, $\hat{c}_{i}$ is fixed at 0 for all items; and for the 1-PAR model, $\hat{c}_{i}$ is fixed at 0 and $\hat{a}_{i}$ is fixed at a constant for all items. 


\section{The Distribution of $Q_{1}$}

It is now argued informally that when the model being examined is true, $Q_{1}$ should be distributed approximately as a chi-square variable with $10-m$ degrees of freedom, where $m$ is the number of item parameters estimated by the latent trait model.

Pearson chi-squares. The general form of the Pearson chi-square statistic is

$$
x^{2}=\sum_{j=1}^{J} \frac{\left(f_{o j}-f_{e j}\right)^{2}}{f_{e j}},
$$

where $f_{o j}$ and $f_{e j}$ are observed and expected frequencies of observations falling in cell $j$. The chisquare distribution is an approximation to the multinomial distribution, and Hays (1973) states:

In the use of the Pearson $\chi^{2}$ statistic to approximate multinomial probabilities, it must be true that:

1. each and every sample observation falls into one and only one category or class interval [cell];

2. the outcomes for the $N$ respective observations in the sample are independent;

3. sample $N$ is large. (p. 722 )

$Q_{1}$ : Observations. In calculating $Q_{1}$, the observations are the item scores for the examinees. Each observation is classified into one of 20 cells based on the value of $\hat{\theta}_{k}$ and on whether the examinee has passed or failed the item. The assumptions are made that (1) the sample size $\sum_{j=1}^{J} N_{j}=N$ is large and that (2) under the null hypothesis (that the model being tested is true), the observations are locally independent (Allen \& Yen, 1979, p. 254).

$Q_{1}$ : Degrees of freedom. The degrees of freedom are calculated as the number of independent data points (i.e., observed proportions) used in $Q_{1}$ minus the number of independent parameters estimated from the data points to produce $E_{i j}$. Because the observed proportion passing an item $\left(O_{i j}\right)$ and the observed proportion failing $\left(1-O_{i j}\right)$ are obviously linearly related, there are at most 10 independent observed proportions in $Q_{1}$. The overall observed proportion passing the item is $\sum_{j=1}^{10} N_{j} O_{i j} / N$ $=P_{i}$; if it is assumed that $P_{i}$ is a fixed value, then one of the $O_{i j}$ is dependent on the other 9 . However, it only makes sense to assume $P_{i}$ is fixed if it is required that $\sum_{j=1}^{10} N_{j} E_{i j} / N=P_{i}$. Because this requirement is not made for the latent trait models, it is assumed that there are 10 independent data points in $Q_{1}$.

Degrees of freedom are subtracted to reflect the extent to which the $E_{i j}$ values are calculated from or are dependent on the values of $O_{i j}$ (Lancaster, 1969, pp. 136, 142-150). $E_{i j}$ is a function of the estimated item parameters and the estimated trait values. The item parameters and trait values are estimated from the examinees' response vectors and not from the $O_{i j}$ values. This means that the estimation process occurs in a sample space that differs from the sample space in which the goodness-offit test is carried out; this can cause a distortion in the chi-square approximation to the distribution of $Q_{1}$ (Kendall \& Stuart, 1967, pp. 428-430; Lancaster, 1969, p. 171). It can be reasoned that because the values of $O_{i j}$ represent fairly well the complete item characteristic function for most items, the item parameter estimates are highly dependent on the values of $O_{i j}$, and one degree of freedom should be subtracted for each estimated item parameter. On the other hand, examinees' trait estimates are based on all the test items, and for long tests each item contributes a small proportion of the information about each $\hat{\theta}_{k}$. Therefore, for any given item there should be a negligible loss of degrees of freedom due to the estimation of the trait values. It should be noted that the determination of the cell boundaries for $Q_{1}$ is dependent on the observed distribution of estimated trait values. This fact may affect the distribution of $Q_{1}$ and its degrees of freedom. 
In any event, the argument is made that when the model being tested is true, $Q_{1}$ will have approximately a chi-square distribution with 10- $m$ degrees of freedom, where there are $m$ item parameters estimated for the latent trait model. The validity of this argument will be examined with simulation data.

\section{The Relationship of $Q_{1}$ to Other Measures of Fit}

Bock's chi-square. Bock (1972) introduced a latent trait model that estimates item parameters for all the answer choices given with the item, not just the correct answer choice. He used a fit measure that examines the suitability of this model. His model can be restricted to deal with only the characteristic function of the correct answer and his fit measure compared to $Q_{1}$. With this restriction, Bock's fit measure equals $Q_{1}$ except for two factors:

1. Examinees are grouped into $J$ cells, but $J$ does not have to equal $10 . J$ does happen to equal 10 in an cxample given in Bóck's paper (pp. 44-45).

2. Bock uses $E_{i j}=P_{i}\left(\hat{\theta}_{\text {med-j }}\right)$, where $\hat{\theta}_{\text {med }-j}$ is the median of the $\hat{\theta}$ values for examinees in cell $j$. If the values of $\hat{\theta}_{k}$ are homogenous within each cell, Bock's method of obtaining $E_{i j}$ will produce a fit measure very similar to $Q_{1}$.

To test the significance of an item's fit, Bock used a chi-square distribution with $J-m$ degrees of freedom, where $m$ independent item parameters have been estimated.

$Q_{2}$. Wright and Panchapakesan (1969) proposed the following fit measure for the 1-PAR model:

$$
O_{2 i}=\sum_{\substack{j=1 \\ N_{j} \neq 0}}^{J} \frac{N_{j}\left(O_{i j}-E_{i j}\right)^{2}}{E_{i j}\left(1-E_{i j}\right)},
$$

where each cell contains examinees with the same number-correct score and examinees with zero and perfect scores are excluded. Because the number-correct score is a sufficient statistic for $\theta$ for the 1PAR model, each cell contains examinees with the same $\hat{\theta}_{k}$. Wright and Panchapakesan stated that the distribution of $Q_{2}$ is approximately chi-square with $J-1$ degrees of freedom, where $J$ cells have $N_{j}$ $\neq 0$. Notice that $Q_{2}$ is very similar to $Q_{1}$, except that $Q_{2}$ involves more cells and constant trait estimates within each cell. $Q_{2}$ was used in selecting items for the KeyMath Diagnostic Arithmetic Test (Connolly, Nachtman, \& Pritchett, 1976) and the Woodcock Reading Mastery Test (Woodcock, 1973). It appears that Whitely and Dawis (1974) used $Q_{2}$ in their evaluation of the 1-PAR model; whereas Rentz and Bashaw (1975) used a "Mean Square Fit," which is $Q_{2}$ divided by the number of score groups with $N_{j} \neq 0$.

$Q_{3}$. The BICAL computer program provided by Wright and Mead (1977) produces another fit measure for the 1-PAR model.

$$
\begin{aligned}
Q_{3 i} & =\frac{1}{J} \sum_{j=1}^{J} \frac{N_{j}^{2}\left(O_{i j}-E_{i j}\right)^{2}}{\sum_{\varepsilon j} \hat{P}_{i}\left(\hat{\theta}_{k}\right)\left[1-\hat{P}_{i}\left(\hat{O}_{k}\right)\right]} \\
& =\frac{1}{J} \sum_{j=1}^{J} \frac{N_{j}\left(O_{i j}-E_{i j}\right)^{2}}{E_{i j}\left(1-E_{i j}\right)-\sigma_{P_{j}}^{2}},
\end{aligned}
$$

Downloaded from the Digital Conservancy at the University of Minnesota, http://purl.umn.edu/93227. 
where

$$
\sigma_{p_{j}}^{2}=\frac{1}{N_{j}} \sum_{k \varepsilon j}^{N_{j}}\left[\hat{P}_{i}\left(\hat{O}_{k}\right)-E_{i j}\right]^{2} .
$$

$\sigma_{P}^{2}$ is the variance within cell $j$ of the predicted proportion passing the item. In calculating $Q_{3}$, examinees are grouped into cells with as close to a uniform distribution as possible, subject to having each number-correct score appear in only one cell, with the recommendation that $N, \geqslant 15$ and with the requirement that $J \leqslant 6$. Notice that $Q_{3}$ is very similar to $Q_{1}$ with the important differences being the number of cells, the subtraction of $\sigma_{P}^{2}$, from the denominator, and the constant $1 / J$.

The denominator of $Q_{3}$ (in the first line of Equation 6) is the variance of the compound binomial distribution (Kendall \& Stuart, 1969, pp. 126-127; Lord \& Novick, 1968, p. 385). It is the theoretical variance of $N, O_{1}$, when the $N_{i}$ examinees in cell $j$ can have different values of $\hat{P}_{2}\left(\hat{\theta}_{k}\right)$. If the variance in values of $\hat{\theta}_{k}$ within cell $j$ is small, and therefore $o_{P}^{2}$ is small, $Q_{1}$ and $J Q_{3}$ will be very similar.

$Q_{4}$. Elliott, Murray, and Saunders (1977) used the following statistic for measuring the fit of the 1-PAR model in choosing items for the British Ability Scales.

$$
Q_{4 i}=\sum_{\substack{j=1 \\ N_{j} E_{i j} \geq 5}}^{J} \frac{N_{j}\left(O_{i j}-E_{i j}\right)^{2}}{E_{i j}} .
$$

Each cell contains examinees with the same number-correct score, with the condition that adjacent cells are pooled until $N, E_{i} \geqslant 5$. Examinees with zero and perfect scores are not included. Elliott et al. (1977) stated that $Q_{4}$ has a chi-square distribution with $J-1$ degrees of freedom, where there are $J$ cells. Notice that $Q_{4}$ will have systematically lower values than $Q_{2} ; Q_{4}$ essentially ignores the item scores that are incorrect (see Equation 1) and violates the requirement that "each and every sample observation falls into one and only one category or class interval [cell]."

\section{Fit Measures Examined in this Paper}

Thus, there are several fit measures that have been used in latent trait research studies that are fairly similar to $Q_{1}, Q_{2}$ and $Q_{4}$ group examinees by number-correct scores, which is suitable for ex amining the 1-PAR model but not suitable for examining the 2-PAR and 3-PAR models. Because this paper examines all three models, it was decided to modify $Q_{2}$ and $Q_{4}$, grouping examinees in the manner that was used for $Q_{1}$. When this is done, $Q_{2}$ becomes essentially equivalent to $Q_{1}$ or $J Q_{3}$, depending on the denominator that is used. It was also decided to use 10 cells for all the fit measures and to delete the constant $1 / J$ from $Q_{3}$. Thus, comparisons would be made among three fit measures, $Q_{1}, Q_{3}^{*}$, and $Q^{*}$, with

$$
Q_{3 i}^{*}=\sum_{j=1}^{10} \frac{N_{j}\left(O_{i j}-E_{i j}\right)^{2}}{E_{i j}\left(1-E_{i j}\right)-\sigma_{P}^{2}},
$$

and

$$
Q_{4 i}^{*}=\sum_{j=1}^{10} \frac{N_{j}\left(O_{i j}-E_{i j}\right)^{2}}{E_{i j}} .
$$




\section{Method}

\section{Simulation Study}

The trait and item parameters that were used in producing simulated response vectors are described as $\theta_{k}, a_{i}, b_{i}$, and $c_{i}$. These values were prespecified and were not estimated from any data set. Trait and item parameters that were estimated from real or simulated response vectors are identified as $\hat{\theta}_{k}, \hat{a}_{i}, \hat{b}_{i}, \hat{c}_{i}$.

Item responses were generated to simulate the performance of students from two adjacent grades. Five hundred trait values $\left(\theta_{k}\right)$ from each of two normal distributions were generated using the IMSL normal random deviate generator GGNML (IMSL, 1979). One $\theta_{k}$ distribution had an observed mean of -.18 and a standard deviation of .95; the other $\theta_{k}$ distribution had an observed mean of .16 and a standard deviation of 1.02. The difference in means for these two groups is typical of the difference between Grades 7 and 8 in performance on the Comprehensive Tests of Basic Skills, Form $S$ (CTB/MicGraw-Hill, 1973). The two trait distributions were pooled to produce one distribution of 1,000 traits. Item discriminations $\left(a_{i}\right)$, item difficulties $\left(b_{i}\right)$, and item lower asymptotes $\left(c_{i}\right)$, were specified to have unimodal, somewhat platykurtic distributions similar to those found with real data. The means and standard deviations of these distributions were $\mu_{a}=1.15, o_{a}=.40, \mu_{b}=-.05, \sigma_{b}=1.15, \mu_{c}$ $=.24$, and $o_{\mathrm{c}}=.04$. (None of the $c_{i}$ values equaled zero.) Parameter sets for 36 items were created by randomly choosing without replacement from the parameter distributions an $a_{i}, b_{i}$, and $c_{i}$ for each item. The resulting correlations of the parameters across items were $r_{a b}=.20, r_{a c}=-.23$, and $r_{b c}=.08$.

For the 3-PAR model the probability of simulee $k$ passing item $i, P_{i}\left(\theta_{k}\right)$, was generated by evaluating the 3-PAR item characteristic function using the prespecified $\theta_{k}, a_{i}, b_{i}$, and $c_{i}$. For each (i.k) pair, a random number from 0 to 1 was generated from a uniform distribution using IMSL subroutine GGUBS (IMSL, 1979). If the random number was less than $P_{i}\left(\theta_{k}\right)$, simulee $k$ was said to pass item $i$; otherwise, simulee $k$ failed item $i$. This item response generation procedure resulted in pass/fail response arrays based on the 3-PAR model for 36 items for 1,000 simulees.

Item responses also were generated using the 2-PAR and 1-PAR models. For the 2-PAR model, $c_{i}$ was set equal to 0 for all items, and then the pass/fail responses were generated for the 36 items and 1,000 simulees. For the 1-PAR model, $c_{i}$ was set equal to 0 and $a_{i}$ was set equal to 1.0 for all items, and then the pass/fail responses were generated. Thus, there were three sets of item responses, one set generated with the 3-PAR model, another set generated with the 2-PAR model, and a third set generated with the 1-PAR model.

Each of the three sets of item responses was analyzed with each of the three latent trait estimating models, producing nine analyses. The item responses were analyzed with the LOGIST computer program (Wood, Wingersky, \& Lord, 1976) to produce estimated trait values $\left(\hat{\theta}_{k}\right)$ and item parameters $\left(\hat{a}_{i}, \hat{b}_{i}, \hat{c}_{i}\right)$. When default options were used, LOGIST automatically estimated traits and item parameters using the 3-PAR model. To estimate traits and item parameters using the 2-PAR model, LOGIST was constrined to hold $\hat{c}_{i}=0$ for all items. To estimate traits and item parameters using the 1-PAR model, LOGIST was constrained to hold $\hat{c}_{i}=0$ and to hold $\hat{a}_{i}$ constant for all items. For all the estimating models, traits were not estimated for simulees with zero or perfect scores.

The fit statistics $-Q_{1}, Q_{3}^{*}$, and $Q^{*}$-were calculated for each item for each of the nine combinations of generating models and estimating models.

\section{Achievement Test Study}

To examine the extent to which the simulation results were helpful in choosing the most appropriate model for real data, eight tests from the Comprehensive Tests of Basic Skills, Form S, Level 3 
(CTB/McGraw-Hill, 1973) were analyzed. The tests were Reading Vocabulary (40 items), Reading Comprehension (45 items), Spelling (30 items), Language Mechanics (20 items), Language Expression (35 items), Mathematics Computation (48 items), Mathematics Concepts ( 25 items), and Mathematics Applications ( 25 items). These are multiple-choice tests with four answer choices per item. Data for 999 seventh- and eighth-grade students were analyzed with the three latent trait models.

\title{
Results
}

\section{Simulation Study}

$Q_{1}$ and $Q_{3}^{*}$ had correlations of 1.0 for each of the nine combinations of generating models and estimating models. Linear regression equations were calculated for predicting $Q^{*}$ from $Q_{1}$ in each of the nine situations. The regression coefficients varied in value from 1.00 to 1.02 and the additive constants varied from -.06 to +.06 . In order to have $Q *$ and $Q_{1}$ be so highly related, $\sigma_{P_{1}}^{2}$ must have been very small. This implies that Bock's fit measure would also have been very similar to $Q_{1}$. Because the results for $Q_{1}$ and $Q_{3}^{*}$ were so similar, only the results for $Q_{1}$ are reported.

Table 1 contains the correlations taken over items of $Q_{1}$ and $Q *$. In general, these two fit statistics led to different conclusions about which items best fit a model. Table 2 contains the means taken over the 36 items of the $Q_{1}$ and $Q *$ statistics. Notice that the $Q^{*}$ statistic tended to be about half the size of the $Q_{1}$ statistic. This result is consistent with the formulas for $Q_{1}$ and $Q^{*}$ (Equations 1 and 10).

The columns of mean fit values have very similar patterns for the data generated by the 3-PAR and by the 2-PAR models. When the data were generated by the 3-PAR model, the 2-PAR estimating model did a surprisingly good job of fitting the data. If a set of real data were being analyzed with all three models, it would be difficult to decide on the basis of the pattern of mean fit values whether the 3-PAR or 2-PAR model was more appropriate. It would be clear that the 1-PAR model was appropriate when the mean fit statistics had similar values for all three estimating models.

Significance tests were conducted to determine whether the fit statistics had chi-square distributions with $10-m$ degrees of freedom. These significance tests were traditional Pearson chi-square tests (Hays, 1973, pp. 725-727) constructed with six cells with equal numbers of fit statistics expected to fall in each cell. The expectations were generated using a chi-square distribution with $10-m$ degrees of freedom, where $m$ item parameters had been estimated by the latent trait model. The significance probabilities of these tests appear in Table 3. (The significance probability is the probability of obtaining a distribution of fit statistics as extreme or more extreme than the observed distribution when the null hypothesis is true, i.e., the fit statistics do have a chi-square distribution with $10-m$ degrees of freedom.)

Given the probabilities in Table 3, it is very unlikely that the $Q *$ statistic has a chi-square distribution. Q* took on values that were too small to be consistent with a chi-square distribution with

\author{
Table 1 \\ Correlations of $Q_{1}$ and $Q_{4}^{*}$ \\ for the Simulation Data
}

\begin{tabular}{|c|c|c|c|}
\hline Estimating & Gene & ating & Mode1 \\
\hline Mode1 & $\overline{3-P A R}$ & $\overline{2-P A R}$ & I-PAR \\
\hline 3-PAR & .82 & .39 & .40 \\
\hline 2-PAR & .84 & .40 & .46 \\
\hline $1-P A R$ & .97 & .94 & .27 \\
\hline
\end{tabular}




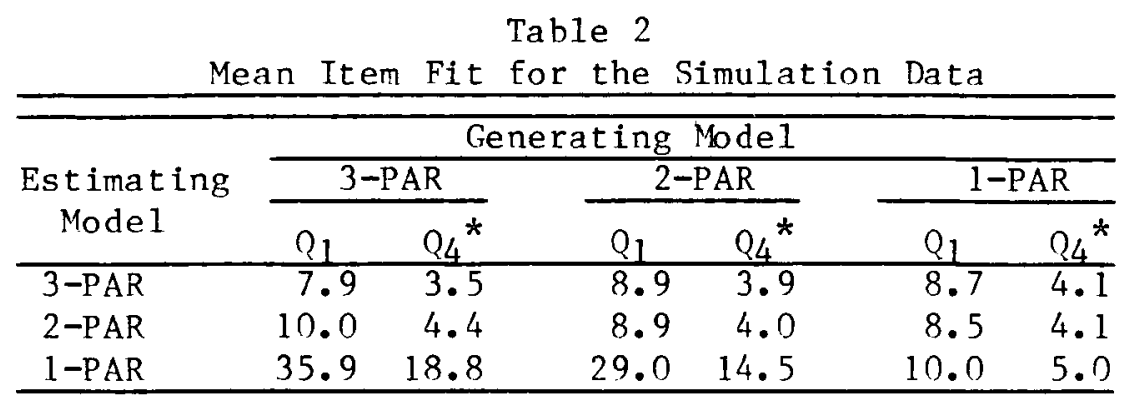

10- $m$ degrees of freedom. On the other hand, it appeared likely that $Q_{1}$ did have a chi-square distribution with $10-m$ degrees of freedom when the null hypothesis was true (i.e., when the estimating model equaled the generating model).

When an estimating model has fewer parameters than the generating model, the significance probability should be very low. This result occurred except when the data were generated by the 3 PAR model and the 2-PAR model was used for estimation. As mentioned earlier, the 2-PAR model did a surprisingly good job of fitting 3-PAR data, and $Q_{1}$ did not detect the fact that the 2-PAR model was not appropriate for the data. Some light can be shed on this finding by examining the relationship between the trait estimates produced by the different models.

When the model used for estimating trait levels and item parameters matched the model used for generating a set of data, the estimated trait levels, item difficulties, and discriminations had strong linear relationships with the trait levels, item difficulties, and discriminating powers in the generating model. In such a situation the lowest correlation of estimated values with the generating values was .89 , which was found for the 3-PAR discriminations. The square root of the mean squared difference between estimated and generating lower asymptotes for the 3-PAR model was .03. In other words, when an estimating model was appropriate, it tended to accurately recover the trait levels and parameters of the generating model. Because of this finding, results are presented only for estimated traits and item parameters. Results involving the generating traits and parameters can be inferred from the results involving the estimated traits and parameters for the estimating model that matches the generating model.

The relationships between trait estimates from the three models are contained in Figure 1. Each column of plots represents the results for one generating model. For each generating model, traits were obtained for three estimating models. The $\left(\begin{array}{l}3 \\ 2\end{array}\right)=3$ pairs of estimated trait values are related in the

Table 3

Significance Probabilities of the Fit

Statistics for the Simulation Data

\begin{tabular}{|c|c|c|c|c|c|c|}
\hline \multirow{3}{*}{$\begin{array}{c}\text { Estimating } \\
\text { Model }\end{array}$} & \multicolumn{6}{|c|}{ Generating Model } \\
\hline & \multicolumn{2}{|c|}{ 3-PAR } & \multicolumn{2}{|c|}{ 2-PAR } & \multicolumn{2}{|c|}{$1-\mathrm{PAR}$} \\
\hline & $Q_{1}$ & $\mathrm{Q}_{4}{ }^{*}$ & $\mathrm{Q}_{1}$ & $\mathrm{Q}_{4}^{*}$ & $Q_{1}$ & $Q_{4}{ }^{\prime}$ \\
\hline 3-PAR & .60 & .00 & .05 & .00 & .25 & .00 \\
\hline $2-P A R$ & .31 & .00 & .25 & .00 & .28 & .00 \\
\hline 1-PAR & .00 & .01 & .00 & .09 & .60 & .00 \\
\hline
\end{tabular}



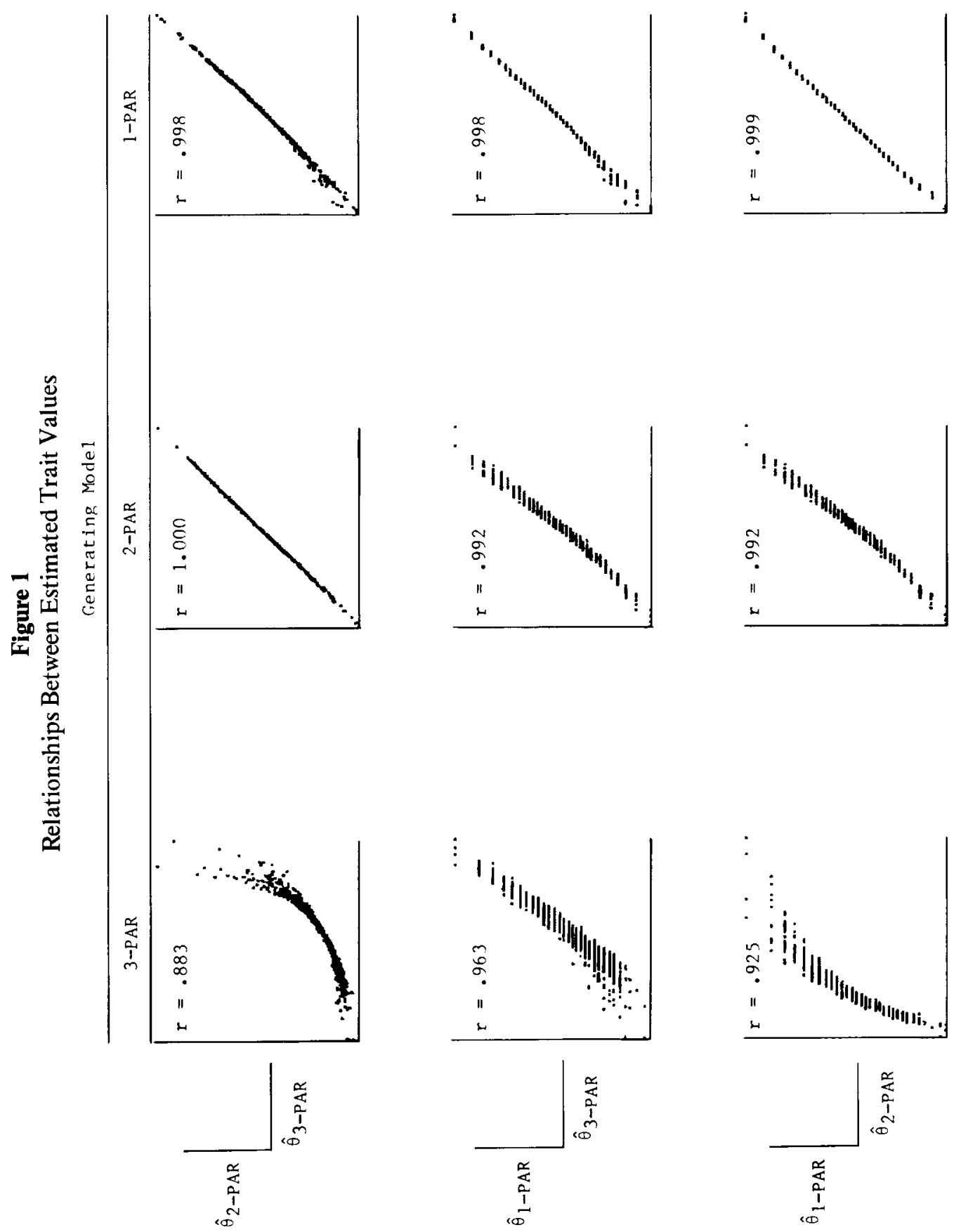
three plots for each generating model, and the plots are labeled in the left margin of the figure. Because the selection of the scale values in the estimation process is arbitrary, numerical labels of the axes of the plots are not important. When data were generated with the 1-PAR or 2-PAR model, every pair of estimated traits was essentially linearly related. When data were generated with the 3-PAR model, pronounced nonlinear relationships between the estimated trait values were created.

An examination of the correlations would not have revealed the curvilinear relationships between the various estimated trait values. Correlation ratios (Hays, 1973, p. 683) were calculated for the $\hat{\theta}$ 's in each of the plots in Figure 1. Two correlation ratios were calculated for each plot, using each of the two sets of $\hat{\theta}$ 's as the independent variable. The "independent" $\hat{\theta}$ 's were grouped in steps of .10 (e.g., $\hat{\theta}$ with values from 1.01 to 1.10 fell in one group). The two correlation ratios for each plot had very similar values, and their average was taken and labelled $\eta^{2}$. The squared correlation was subtracted from the average correlation ratio for each plot. This difference indicates the proportion of variance shared by the two variables beyond the variance shared in a linear relationship. This difference was greater than .05 for two of the plots:

1. When data were generated with the 3-PAR model and traits estimated by the 3-PAR and 2-PAR models were compared, $\tilde{\eta}^{2}-r^{2}=.19$.

2. When data were generated by the 3-PAR model and traits were estimated by the 1-PAR and 2PAR models, $r^{2}-r^{2}=.10$.

These differences between $\eta^{2}$ and $r^{2}$ quantitatively verify the existence of the obvious curvilinear relationships in Figure 1.

Figure 2 displays the relationships between the item difficulties estimated by the three models. The pattern of these relationships parallels the pattern found for the traits; but because there are fewer items than examinees, the patterns are more difficult to see with the items than with the trait values.

For data generated with the 2-PAR and 3-PAR models, the relationships between the estimated item discriminations and the relationships between item discriminations and item difficulties are contained in Figure 3. The relationships between the 3-PAR and 2-PAR estimated item discriminations were dramatically different, depending on whether the data were generated with the 3-PAR or 2-PAR model. When the 3-PAR model generated the data, the 3-PAR and 2-PAR estimated discriminations had a weak relationship. When the 2-PAR model generated the data, the 3-PAR and 2-PAR estimated discriminations had a very strong linear relationship.

When the 3-PAR model was used for estimation, there was a low positive correlation between $\hat{a}_{i}$ and $\hat{b}_{i}$ regardless of which model generated the data. This low positive correlation was consistent with the low positive correlation between $a_{i}$ and $b_{i}$ for the item parameters used in the data generation. When the 2-PAR model was used for estimation, there was a substantial difference in the correlation between $\hat{a}_{i}$ and $\hat{b}_{i}$ found when the 2-PAR model generated the data versus when the 3-PAR model generated the data. If the 2-PAR model generated the data, the low positive correlation between $\hat{a}_{i}$ and $\hat{b}_{i}$ appeared. However, if the 3-PAR model generated the data, use of the 2-PAR model for estimation produced a strong negative correlation between $\hat{a}_{i}$ and $\hat{b}_{i}$.

\section{Achievement Test Study}

To examine how similar the simulation data were to the Comprehensive Tests of Basic Skills (CTBS) data, means and standard deviations of estimated item parameters were compared. When the 3-PAR model generated the simulation data and was used in the estimation, the mean $\hat{a}=$ was 1.39 , with $S D$ of .57 ; mean $\hat{b}=-.02, S D=1.08$; and mean $\hat{c}=.23, S D=.01$. These estimates were obtained with estimated traits with a mean of zero and a standard deviation of one. When the 3-PAR es- 

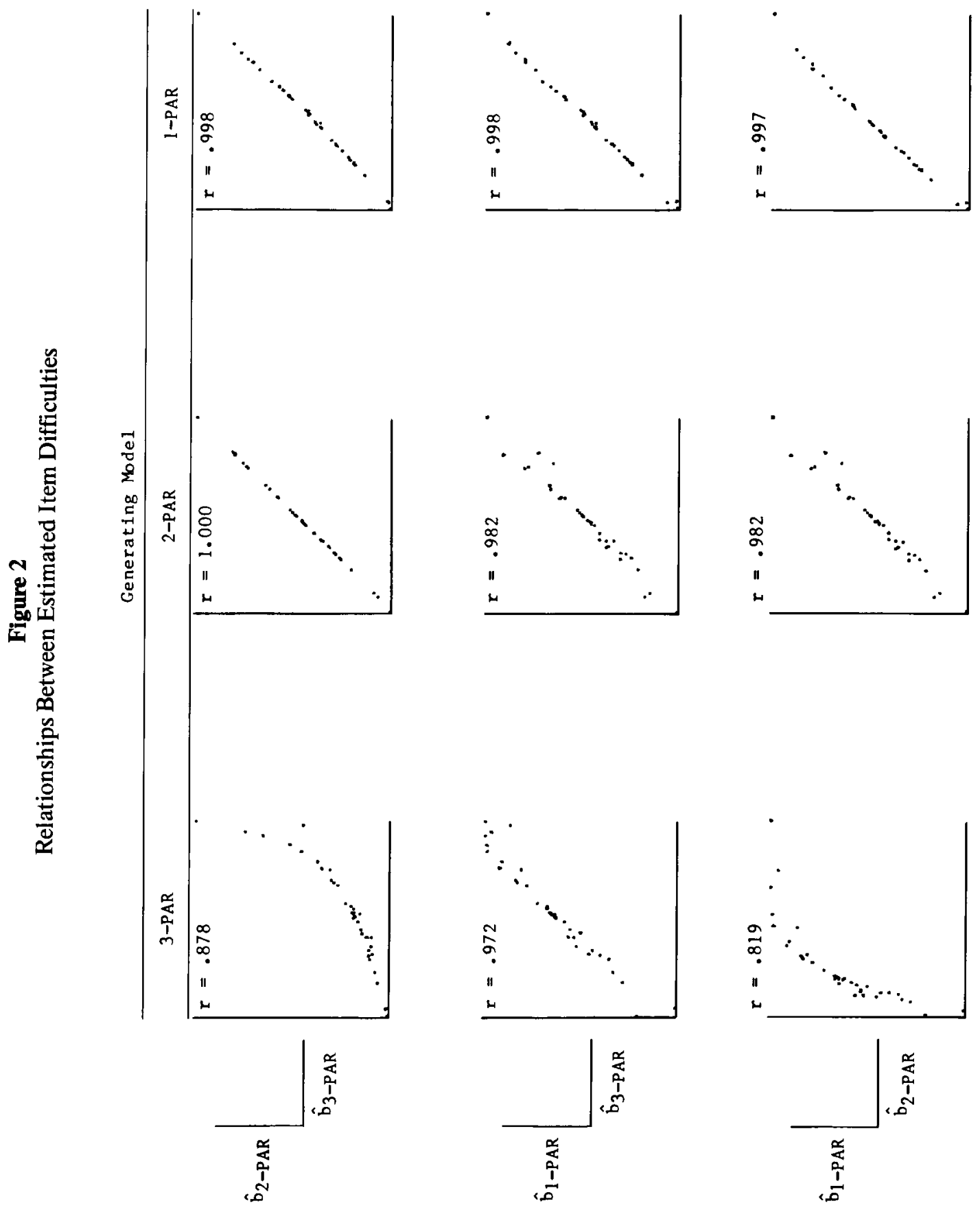

Downloaded from the Digital Conservancy at the University of Minnesota, http://purl.umn.edu/93227. requires payment of royalties through the Copyright Clearance Center, http://www.copyright.com/ 
Figure 3

Relationships Between Estimated Item Discriminations

Across Different Estimating Models

and Between Estimated Item Discriminations and Item Difficulties

Within One Estimating Model
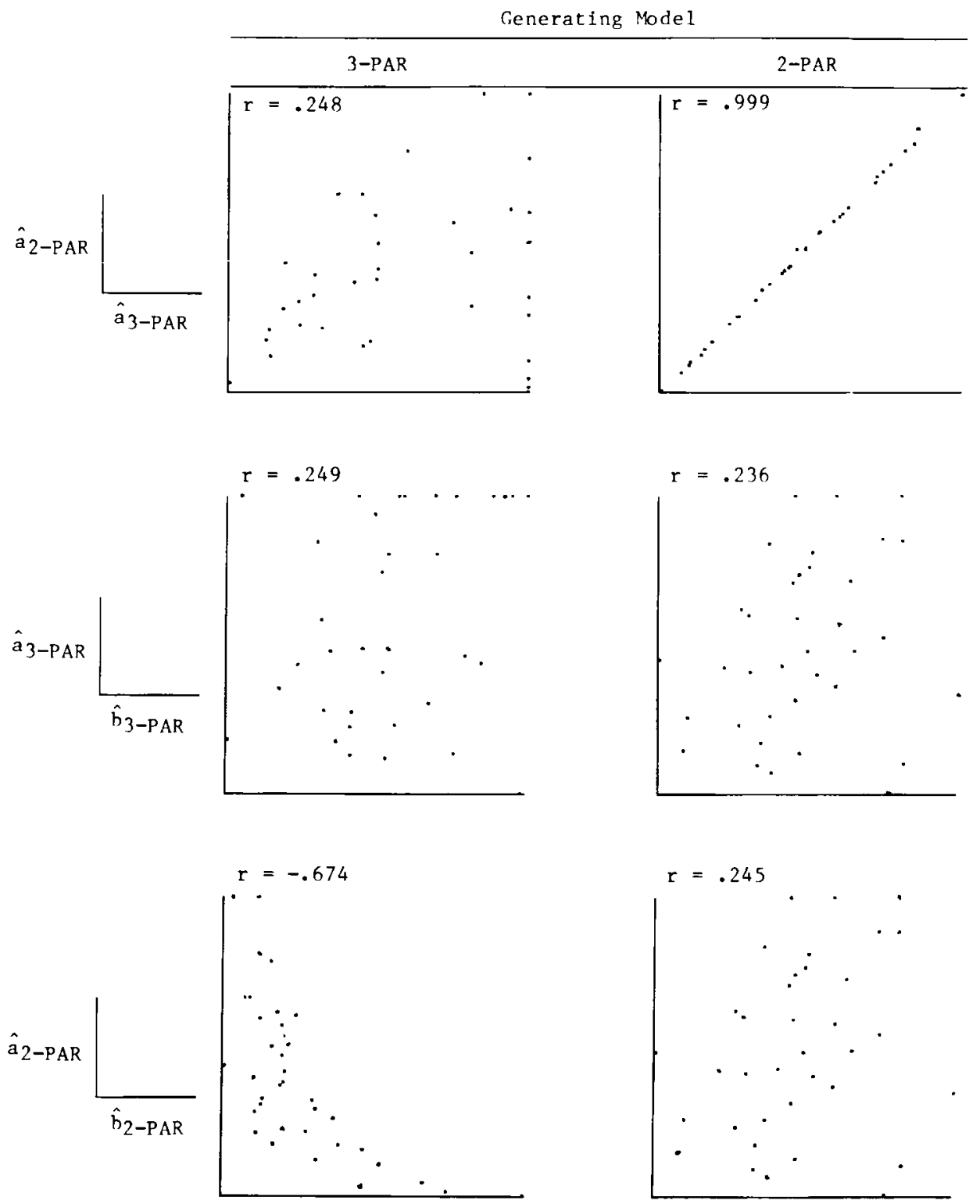

timating model was used for the CTBS data and the estimated traits had a mean of zero and a standard deviation of one, the range of item parameter statistics were mean $\hat{a}$ of 1.18 to 1.68 , with $S D$ of .55 to .80 ; mean $\hat{b}$ of -.08 to $.15, S D$ of .54 to .75 ; mean $\hat{c}$ of .17 to .23 , with $S D$ of .01 to .03 . The 
Table 4

Mean Values of $Q_{1}$ for the CTBS Tests

\begin{tabular}{cllrrrrrr}
\hline $\begin{array}{c}\text { Estimating } \\
\text { Mode1 }\end{array}$ & $\begin{array}{l}\text { Read. } \\
\text { Vocab. }\end{array}$ & $\begin{array}{l}\text { Read. } \\
\text { Comp. }\end{array}$ & Spel1. & $\begin{array}{l}\text { Lang. } \\
\text { Mech. }\end{array}$ & $\begin{array}{l}\text { Lang. } \\
\text { Exp. }\end{array}$ & $\begin{array}{l}\text { Math. } \\
\text { Comp. }\end{array}$ & $\begin{array}{r}\text { Math. } \\
\text { Conc. App1ic. }\end{array}$ \\
\hline 3-PAR & 11.9 & 11.4 & 8.5 & 14.5 & 9.5 & 12.5 & 11.3 & 9.8 \\
2-PAR & 14.4 & 13.0 & 8.9 & 12.2 & 10.6 & 14.7 & 12.6 & 12.1 \\
1-PAR & 30.7 & 32.4 & 24.3 & 27.2 & 29.4 & 26.3 & 24.6 & 25.8 \\
\hline
\end{tabular}

simulation data, therefore, appeared to be similar to the CTBS data except that the simulation data had items that tended to have a greater variance in item difficulties than did the CTBS data.

The mean values of $Q_{1}$ for the three latent trait models for each of the eight CTBS tests are contained in Table 4. These means have higher values but similar patterns to those in Table 2 for the simulation data generated with the 3-PAR and 2-PAR models. For every test except Spelling and Mathematics Applications, the significance probabilities of the $Q_{1}$ statistics were less than .05 for all three models. The estimated trait values and item parameters for each CTBS test were examined, and their relationships appeared very similar to the relationships found for the simulation data generated with the 3-PAR model. For example, Table 5 contains the correlations between the estimated item difficulties and discriminations for the 3-PAR and 2-PAR models. These correlations are similar in pattern to the correlations found with simulation data generated with the 3-PAR model.

All the results for Reading Vocabulary, which were typical of the results for the other tests, are contained in Figure 4. The $\hat{\theta}$ plots in the first column of Figure 4 can be compared with each of the columns of plots in Figure 1; the Reading Vocabulary trait estimates appear most similar to the trait estimates based on simulation data generated with the 3-PAR model. The $\hat{b}_{1}$ plots in the second column of Figure 4 can be compared with each of the columns of plots in Figure 2; it is not clear which data generation model is most consistent with the results for the Reading Vocabulary item difficulties. The column of $\hat{a}_{i}$ plots in Figure 4 can be compared to the two columns of plots in Figure 3; the estimated item discriminations for the Reading Vocabulary data clearly are most consistent with the results for the simulation data generated with the 3-PAR model.

\section{Discussion}

\section{Behavior of Fit Measures with Simulated Data}

For most of the sets of simulated data, $Q *$ and $Q_{1}$ had only moderate correlations; and for all sets of data, $Q^{*}$ took on values that were about half the size of the values of $Q_{1}$. When an estimating model was true, the values of $Q *$ were systematically too small for $Q *$ to be distributed as a chisquare variable with $10-m$ degrees of freedom. The statistics $Q_{1}$ and $Q_{3}^{*}$ produced nearly identical re-

Table 5

Correlations Between Estimated Item Discriminating Powers and Estimated Item Difficulties for the CTBS Tests

\begin{tabular}{|c|c|c|c|c|c|c|c|c|}
\hline $\begin{array}{l}\text { Estimating } \\
\text { Model }\end{array}$ & $\begin{array}{l}\text { Read. } \\
\text { Vocab. }\end{array}$ & $\begin{array}{l}\text { Read. } \\
\text { Comp. }\end{array}$ & & $\begin{array}{l}\text { Lang. } \\
\text { Mech. }\end{array}$ & $\begin{array}{l}\text { Lang. } \\
\text { Exp. }\end{array}$ & $\begin{array}{l}\text { Math. } \\
\text { Comp. }\end{array}$ & $\begin{array}{l}\text { Math. } \\
\text { Conc. }\end{array}$ & $\begin{array}{l}\text { Math. } \\
\text { Applic. }\end{array}$ \\
\hline 3-PAR & .53 & .33 & -.22 & .56 & -.18 & .58 & .41 & .40 \\
\hline 2-PAR & -.33 & -.41 & -.57 & .23 & -.47 & .23 & -.44 & -.45 \\
\hline
\end{tabular}




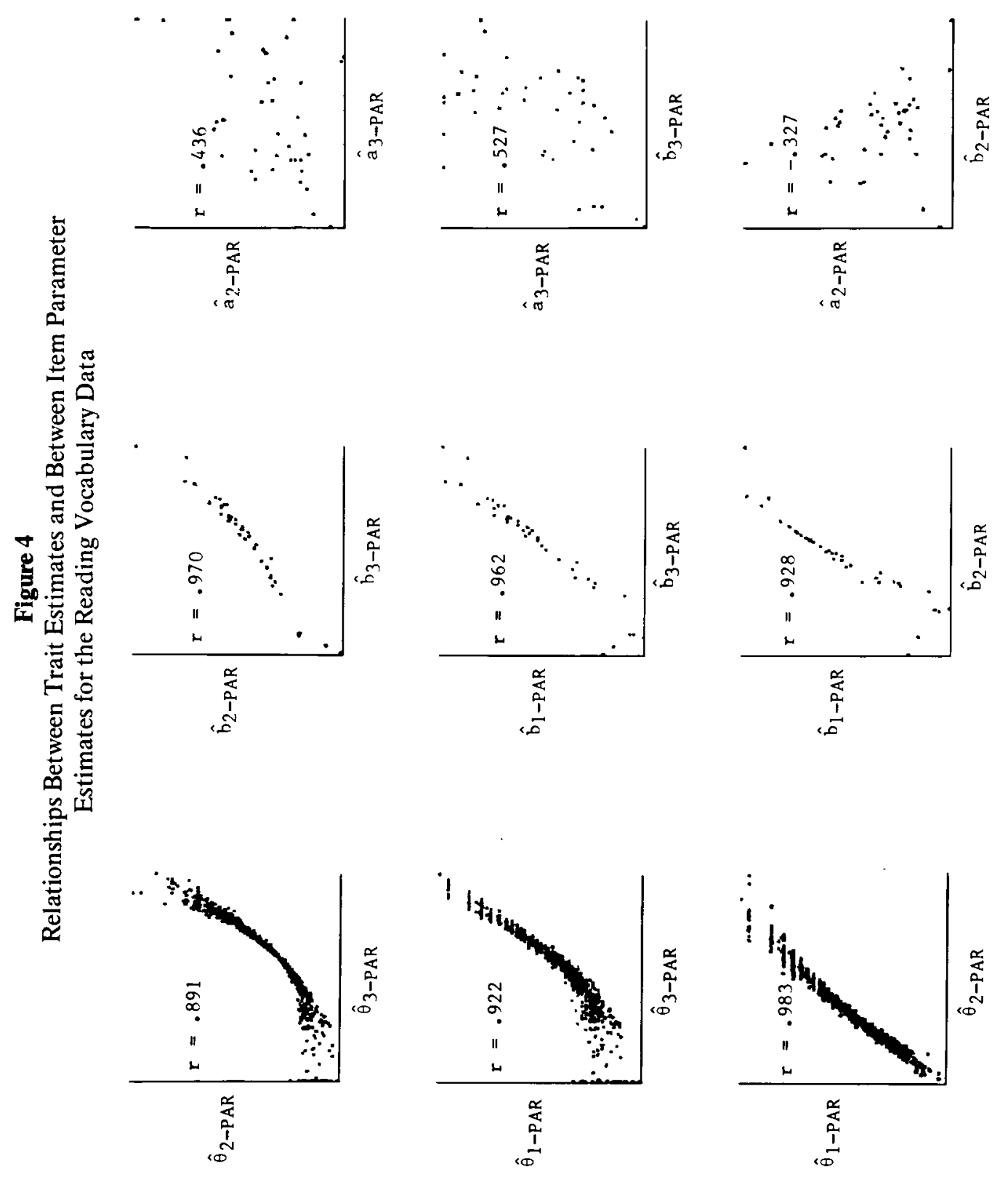


sults for all nine sets of simulation data, with the values of $Q *$ tending to be only slightly larger than the values of $Q_{1}$. Thus, for the type of data sets examined, which simulated the pooling of examinees from two adjacent grades, the use of 10 cells was sufficient to produce relatively small values of $\sigma_{P_{j}}^{2}$ and small within-cell variances in $\hat{\theta}_{k}$. This result implies that with 10 cells Bock's (1972) fit measure would also be nearly identical to $Q_{1}$ for data sets with similar amounts of variance in $\hat{\theta}_{k}$.

When an estimating model matched the generating model, the hypothesis that $Q_{1}$ had a chisquare distribution with $10-m$ degrees of freedom would never have been rejected at the .05 level. However, for these data sets the mean value of $Q_{1}$ was always greater than $10-m$, indicating that perhaps too many degrees of freedom were subtracted for the estimated parameters. ${ }^{1}$ In any case, it appears reasonable to describe $Q_{1}$ as having approximately a chi-square distribution with $10-m$ degrees of freedom when (1) the null hypothesis is true, (2) $N \doteq 1000$, and (3) 36 items are used. The exact distribution of $Q_{1}$ has not been specified. The most important information about $Q_{1}$ that was gained from the simulation study was the pattern of mean values for the nine combinations of generating and estimating models (Table 2). It is this pattern of the mean values of $Q_{1}$ that is useful in interpreting results for real data.

It was not a goal of this study to describe the behavior of $Q_{1}$ for a wide variety of conditions but to describe its behavior for a set of conditions similar to the conditions existing for the real achievement test data. The number of cells and the number and distribution of examinees are variables that can be expected to influence the value of $Q_{1}$, but the simulation was structured to be equal or very similar to the real data with respect to these variables. The number of items and the values of the item parameters can be expected to influence $Q_{1}$, but the items in the simulation study were similar to the items analyzed for the real data. The fact that the pattern of results for the simulation data generated with the 3-PAR model so closely paralleled the patterns of results for all eight achievement tests supports the contention that the simulated data were successful in modeling the real data.

\section{Inappropriate Use of a Model}

The most surprising simulation results appeared when data that were generated with the 3-PAR model were analyzed with the 2-PAR model:

1. The 2-PAR model fit the data almost as well as the 3-PAR model fit the data (Table 2).

2. The traits (and item difficulties) as estimated by the 2-PAR and 3-PAR models were nonlinearly related, with the 2-PAR trait estimates more stretched out at the high end than the 3-PAR traits (Figure 1).

3. The 2-PAR and 3-PAR estimated item discriminations had a weak relationship (Figure 3).

4. The 2-PAR estimated item difficulties and discriminations displayed a strong negative correlation (Figure 3).

The explanation of these results centers around the fact that the 2-PAR model with $c_{i}=0$ attempts to fit or explain item characteristic functions with nonzero asymptotes. It is most awkward for the 2-PAR model to explain a nonzero asymptote when an item is difficult and has a moderate to high discrimination. When an item is easy, there is little data available to establish the item's lower asymptote. When an item has a low discrimination, the nonzero asymptote can be fairly well explained solely on the basis of a low discrimination. The 2-PAR model deals with the difficult, highly discriminating items with nonzero asymptotes by stretching out the trait (and difficulty) scale at the high end. This stretching decreases the slope of the item characteristic curves of difficult items, and the result-

'The expected value of a chi-square variable is its degrees of freedom. 
ing item characteristic curves can be explained with low discriminations.

Thus, although the 2-PAR model is almost as accurate as the 3-PAR model in explaining pass/fail response arrays generated by a 3-PAR model, the 2-PAR model produces trait estimates and item parameters that are different from the trait levels and parameters used in generating the data. Because an item's discrimination can be affected by the item's difficulty level relative to the ability level of the group of examinees, the 2-PAR estimation of discriminations can be sample dependent for 3-PAR data. Difficult items can have systematically lower discriminations if they happen to be calibrated using a group of examinees with a relatively low ability level than if they happen to be calibrated with relatively able examinees. This sample dependency means that the predictions of the 2PAR model can be accurate for the sample in which the 2-PAR item parameters are estimated, but the predictions for some items can be inaccurate in cross-validations involving samples of examinees that differ systematically from the sample in which the parameters were estimated. These problems with the 2-PAR estimating model can occur when the data are generated with the 3-PAR model; the 2-PAR estimating model works well with data generated with 2-PAR or 1-PAR models.

When the simulation data were generated by the 3-PAR model, the 1-PAR estimating model produced trait estimates that were fairly linearly related to the 3-PAR trait estimates (Figure 1). Because $r_{a b}$ was fairly close to zero for the 3-PAR generating model, the 1-PAR model (with $r_{a b}$ constrained to equal zero) produced a trait scale similar to the 3-PAR trait scale. However, if $r_{a b}$ had been strongly positive or negative for the 3-PAR generating model, it would be expected that the 1-PAR estimating model would have produced a trait scale with a more pronounced curvilinear relationship with the 3PAR trait scale. Indeed, such a relationship occurred with the Reading Vocabulary data where $r_{\hat{a} \hat{b}}=$ .53 for the 3-PAR model (Figure 4). In a similar fashion, when data were generated by the 2-PAR model, the 1-PAR and 2-PAR trait estimates were linearly related for the most part. However, if $r_{\dot{a} \dot{b}}$ had been strongly positive or negative for the 2-PAR generating model, it would be expected that the 1-PAR and 2-PAR trait estimates would have shown a pronounced curvilinear relationship.

There are several practical problems that can arise when a 2-PAR or 1-PAR model is used inappropriately. One problem is the sample dependency of some item parameter estimates that can occur with the 2-PAR model. If item parameter estimates are sample dependent, one of the major theoretical advantages of the use of the models is lost. Another problem is inaccurate model predictions. When a 1-PAR model is used with multiple-choice items, the model predictions can be expected to be much less accurate (as measured by $Q_{1}$ ) than the 2-PAR or 3-PAR model predictions. This inaccuracy can be a problem if, for example, the model is being used to predict the probability that certain examinees will pass items they did not take.

A third problem is the lack of a perfect correlation between the estimated trait values and actual trait values when the estimating model is inappropriate. The correlations between the different trait estimates are high but not perfect. Examinees will receive somewhat different rank orderings or percentiles when different models are used. The nonlinear relationships between the various trait estimates implies that they can produce different results in correlational studies and different results when comparing, for example, group means and growth.

Another problem arising with the inappropriate use of a 1-PAR model is discussed by Gustafsson (1979):

The Rasch model can be used successfully to solve the problem of vertical equating, as long as there is no correlation between item discrimination and difficulty. When there is a positive or negative correlation between these two parameters, there will be a bias [in the equating], with the direction of bias being dependent upon the sign of the correlation. . . In [multiple-choice] tests there tends to be a negative correlation between item discrimination and item difficulty, if a pa- 
rameter representing the lower asymptote of the item characteristic curve is not estimated. (p. 157)

An inaccurate vertical equating can be a serious problem in many testing situations.

There is another potential problem with the use of the 1-PAR model with data that are actually consistent with the 2-PAR or 3-PAR model. One of the assumptions of the 1-PAR model is that item discriminations are constant over the items. When the 1-PAR model is fit to a set of data, it chooses a trait scale that tends to minimize the correlation between item discriminations and difficulties. This choice of the scale is not in itself problematical and can actually be seen as desirable. However, a problem can arise when the 1-PAR model is used to equate scores on two tests if in one of these tests the correlation between item difficulty and discrimination (as measured by the 2-PAR model) is positive and in the other test this correlation is zero or negative. In this situation, the 1-PAR model will produce trait scales that are nonlinearly related for the two tests. Such a nonlinear relationship can be detected (e.g., by examining plots of trait values for examinees who take both tests) and the linear equating not used.

This study does not examine the problems involved in the inappropriate use of the 3-PAR model. Using $Q_{1}$, the 3-PAR model would have been rejected at the .05 level for all but two of the achievement tests. The rejection of the model is not too surprising because it would not be expected that any model would be strictly true in describing complex test-taking behavior and it would be expected that a sample size of about 1,000 would be large enough to make $Q_{1}$ a fairly powerful fit statistic. However, the practical implications of the inaccuracies of the 3-PAR model remain to be defined.

\section{Choosing A Model For Real Data}

The simulation results were the foundation for a two-step procedure for choosing the most appropriate of the three latent trait models for a set of real data.

Step 1: Compare mean values of $Q_{1}$ for all three models. If these three values are approximately equal, the 1-PAR model is appropriate. If the mean value of $Q_{1}$ for the 1-PAR model is substantially greater than the mean values for the other models, then the 1-PAR model is not appropriate. Proceed to Step 2 to determine whether the 2-PAR or 3-PAR model is more appropriate.

Step 2: The simulation results lead to the expectation that the mean values of $Q_{1}$ for the 2-PAR and 3-

PAR models will tend to be similar and these mean values will not help choose which of these two models is more appropriate. Instead, it is helpful to examine the relationships between the trait estimates and item parameters for the models. If the 2-PAR and 3-PAR trait estimates are nonlinearly related, the 2-PAR and 3-PAR item discriminations have a low correlation, and the correlation between item difficulties and item discriminations is substantially lower for the 2 PAR model than for the 3-PAR model, then the 3-PAR model is more appropriate. If, however, the 2-PAR and 3-PAR trait estimates are highly linearly related, the 2-PAR and 3-PAR item discriminations are highly linearly related, and the correlation between item difficulties and item discriminations is very similar for the 2-PAR and 3-PAR models, then the 2-PAR model can be used.

When this procedure was followed for the eight achievement tests, it was clear that the 3-PAR model was the most appropriate of the three models to use for every test. The two-step procedure was far more helpful than examining the significance probabilities of the $Q_{1}$ values for the three models.

A test user may wish to choose one of the three latent trait models a priori and not go to the trouble or expense of examining data with all three models. The results obtained here for the eight achievement tests indicate that it is likely that it will not be appropriate to use the 2-PAR or 1-PAR 
models with multiple-choice tests and that the 3-PAR model should be the a priori choice. However, use of the 3-PAR model requires larger sample sizes for accurate item parameter estimates, more computer time to obtain those estimates, and more technical sophistication than the use of the 2-PAR or 1-PAR model, and many multiple-choice test users choose a priori the 1-PAR model. If a multiplechoice test user chooses a 1-PAR or 2-PAR model, the user should be prepared to demonstrate the appropriateness of the model or, failing that, to deal with possible practical problems arising from the use of an inappropriate model, as described in the previous section.

\section{References}

Allen, M. J., \& Yen, W. M. Introduction to measurement theory. Monterey, CA: Brooks/Cole, 1979.

Bock, R. D. Estimating item parameters and latent ability when responses are scored in two or more nominal categories. Psychometrika, 1972, 37. 29-51.

Connolly, A. J., Nachtman, W., \& Pritchett, E. M. KeyMath Diagnostic Arithmetic Test manual. Circle Pines, MN: American Guidance Service, 1976.

CTB/McGraw-Hill. Comprehensive Tests of Basic Skills, Form S. Monterey, CA: Author, 1973.

Elliott, C. D., Murray, D. J., \& Saunders, R. Good ness of fit to the Rasch model as a criterion of test unidimensionality. Manchester: University of Manchester, 1977.

Gustafsson, J. E. The Rasch model in vertical equating of tests: A critique of Slinde and Linn. Journal of Educational Measurement. 1979, 16. 153-158.

Hays, W. L. Statistics for the social sciences. San Francisco: Holt, Rinehart, \& Winston, 1973.

IMSL Library (7th ed.). Houston, TX: International Mathematical and Statistical Libraries, 1979.

Kendall, M. G., \& Stuart, A. The advanced theory of statistics (Vol. 2). London: Griffin, 1967.

Kendall, M. G., \& Stuart, A. The advanced theory of statistics (Vol. 1). London: Griffin, 1969.

Lancaster, H. O. The chi-squared distribution. New York: Wiley, 1969.

Lord, F. M., \& Novick, M. R. Statistical theories of mental test scores. Menlo Park, CA: AddisonWesley, 1968.
Rentz, R. R., \& Bashaw, W. L. Equating reading tests with the Rasch model: Final report. Athens, GA: University of Georgia, Educational Research Laboratory, September $\mathbf{i} \overline{9} \overline{7}$.

Whitely, S. E., \& Dawis, R. V. The nature of objectivity with the Rasch model. Journal of Educational Measurement. 1974, 11, 163-178.

Wood, R. L., Wingersky, M. S., \& Lord, F. M. LOGIST: A computer program for estimating examinee ability and item characteristic curve parameters (RM-76-6). Princeton, NJ: Educational Testing Service, 1976.

Woodcock, R. W. Woodcock Reading Mastery Tests manual. Circle Pines, MN: American Guidance Service, 1973.

Wright, B. D., \& Mead, R. J. BICAL: Calibrating items and scales with the Rasch model (Research Memorandum No. 23). Chicago, IL: University of Chicago, Department of Education, Statistical Laboratory, 1977.

Wright, B., \& Panchapakesan, N. A procedure for sample-free item analysis. Educational and Psychological Measurement. 1969, 29. 23-48.

\section{Author's Address}

Send requests for reprints or further information to Wendy M. Yen, CTB/McGraw-Hill, Del Monte Research Park, Monterey, CA 93940. 